Le Devoir, Montréal, Mardi, le 29 juillet 2003, page A7 - idées

\title{
LE DEVE⿺
}

\section{La mini-ministérielle de l'OMC à Montréal}

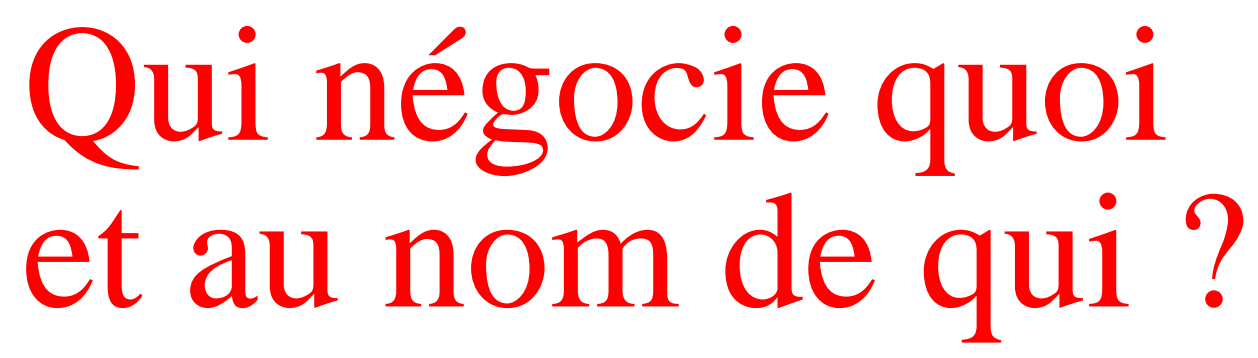

Dorval Brunelle

Directeur du Groupe de recherche sur l'intégration continentale (GRIC/UQAM) ; auteur de Dérive globale (Boréal, 2003)

Normand Pépin

Responsable du service de la recherche, Centrale des syndicats démocratiques (CSD)

L'initiative du ministre du Commerce international, Pierre Pettigrew, de tenir à Montréal, du 28 au 30 juillet 2003, une « Miniministérielle informelle » réunissant quelque 25 ministres du Commerce de pays membres de l'Organisation mondiale du commerce (OMC), permet de mettre en évidence les grandes failles du régime actuel de négociations commerciales à l'échelle mondiale.

Dès sa création en 1995, l'OMC hérite d'un problème double. Le premier est de savoir comment lier entre elles les négociations conduites jusque-là dans le cadre d'accords séparés et facultatifs portant sur les biens (GATT), sur les services
(GATS/AGCS), sur les investissements (TRIMS) et les droits de propriété intellectuelle (TRIPS/ADPICS). Le second : comment et sur quelles bases engager des négociations entre des partenaires aussi disparates et inégaux, qui sont au nombre de 146 à l'heure actuelle ?

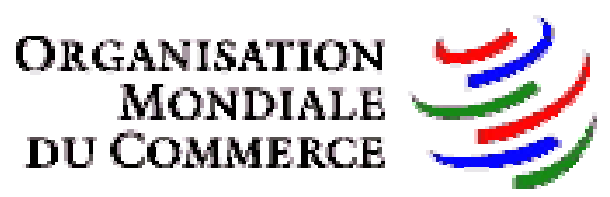

Pour faire face au premier défi, on convient d'appliquer les mêmes principes, en l'occurrence, le traitement national et la clause de la nation la plus favorisée dans tous les domaines, et ce, peu importe s'il s'agit de biens, de services, d'agriculture ou d'investissements. La justification qui est fournie à ce propos est qu'il faut désormais sortir d'un régime d'exception qui a conduit à la multiplication des entraves au commerce mondial au lieu de les réduire. Pour faire face au deuxième défi, les décisions ne sont pas prises à la majorité des membres, elles reposent sur la recherche des 
consensus. La justification qui est fournie cette fois-ci est que la recherche des consensus offre plus de souplesse dans les négociations.

\section{Réponses dommageables}

Or, les deux réponses apportées à ces deux défis sont non seulement mauvaises, elles sont dommageables pour deux raisons.

Premièrement, le principe d'universalité est porteur des pires injustices, à moins que les rigueurs de son application ne soient tempérées par le recours à un autre principe, le principe de différenciation. Il est donc inacceptable, tant du point de vue théorique que du point de vue pratique, que l'on ait renoncé à prévoir quelques principes de différenciation qui permettraient de faire droit au fait que tous les biens, tous les services, tous les investissements etc., ne sont pas commercialisables partout, toujours, pour tous, dans tous les cas, et que, si on ne veut pas créer de nouvelles injustices, il faut cerner et définir ces différenciations afin de réduire les écarts au lieu de les accroître. Pour le moment, le travail de réflexion en cours sur ce sujet à l'OMC est nettement insuffisant.

Deuxièmement, le recours au consensus ne peut ni ne doit remplacer le démocratisme, car c'est bien la démocratie qui, en dernière instance, permet non seulement d'établir la légalité et la légitimité d'un principe ou d'une norme, mais également d'atténuer les effets pervers des regroupements informels qui ne manquent jamais de se constituer autour d'enjeux aussi litigieux quand le flou l'emporte sur la définition de procédures transparentes. Pour le moment, le rôle des groupes informels à l'OMC est déplorable.
On voit alors que la convocation d'une mini-ministérielle reflète le double blocage dont il vient d'être question. Quant au contenu, on sait maintenant que les grands engagements souscrits à Doha ne peuvent pas être respectés pour deux raisons : d'abord, à cause du protectionnisme appliqué par les pays riches, et par les membres du QUAD (un groupe informel constitué des États-Unis, de l'Union européenne, du Japon et du Canada) en particulier; ensuite, à cause des difficultés que rencontrent les pays moins développés à mettre en oeuvre les engagements auxquels ils ont souscrits. Quant au protocole, on peut en toute légitimité se demander qu'est-ce que 25 pays peuvent bien faire à négocier entre eux, alors qu'ils constituent un peu plus du sixième des membres de l'organisation?

\section{Bien commun}

Le gouvernement canadien a toujours cherché à défendre l'approche multilatérale, et ce, même dans la situation difficile que nous avons connue l'hiver dernier à l'ONU, de sorte qu'il n'y a pas de raison valable pour justifier que la préparation de la 5e Conférence ministérielle de l'OMC, qui doit se dérouler à Cancún, au Mexique, du 10 au 14 septembre prochain, échappe à cette règle.

En attendant, comme le gouvernement canadien a exprimé à plusieurs reprises son opposition à une application universelle des principes du droit commercial à certains services, notamment à l'éducation, à la santé et à la diversité culturelle, il faut de toute nécessité que cette position soit réaffirmée et renforcée afin qu'elle puisse recueillir de nouveaux appuis parmi les partenaires de l'OMC.

Ensuite, les accords en cours de négociations, (OMC, ZLEA, et autres 
accords bilatéraux) universalisent la commercialisation privée de tous les produits, services, investissements et droits de propriété sans égards à leurs finalités sociales, environnementales et politiques. Ce faisant, ces négociations favorisent systématiquement les secteurs privés de production et de distribution au détriment des secteurs publics, communautaires et sociaux, ce qui conduit à l'établissement de véritables chartes de protection des droits des investisseurs au détriment de tous les autres acteurs économiques.

En effet, les négociations de l'OMC, comme celles de la zone de libre-échange des Amériques (ZLEA), et à l'instar de ce qui existe déjà dans l'ALENA, font la part belle aux investisseurs privés en leur accordant des droits excessifs, sous prétexte qu'il faut à tout prix protéger l'investissement étranger. Il fut un temps où le ministre Pettigrew avait lui-même exprimé de fortes réserves face à de telles dispositions et il serait souhaitable que ces réserves reprennent leur place dans les discussions.

Enfin, à titre de ministre et de parlementaire, l'hôte de cette réunion porte la responsabilité de défendre et de promouvoir le bien commun, et non pas le bien de quelques-uns. À nos yeux, la défense et la promotion du bien commun exigent l'application des principes de justice et de développement à visage humain qui avaient été incorporés à la Déclaration du Sommet des peuples adoptée à Québec en avril 2001. La Déclaration prévoyait, entre autres choses, qu'il fallait « assurer le fonctionnement et l'application de mécanismes démocratiques dans les consultations à chacune des étapes des négociations en cours au sein de l'OMC comme de la ZLEA; agir dans le respect de la primauté des libertés et des droits individuels et collectifs sur le commerce et le profit; et, accroître de manière substantielle l'aide publique au développement et favoriser le développement durable plutôt que de diriger les surplus budgétaires vers la sécurité, la guerre et la restriction des droits ».

Ces valeurs ont été ignorées à l'OMC et l'organisation court vers un échec à Cancún. C'est ce qui explique la tenue de cette «mini-ministérielle» à Montréal. L'urgence d'agir est là. Il reste à voir si la volonté politique des économies les plus puissantes sera à la hauteur des défis.

$$
* * *
$$

La diffusion de cet article a reçu l'aval du Réseau québécois sur l'intégration continentale (RQIC), dont le GRIC et la CSD sont membres, ainsi que du Conseil des Canadiens. 